\title{
Pathologic Q Waves and Prolonged QTc Time in Preoperative ECG Are Predictive for Perioperative Cardiovascular Events
}

\author{
Thomas Wolters ${ }^{1,2 *}$, Michael Weber ${ }^{3,4}$, Thomas Neumann³, Hans-Georg Strauß1, \\ Andreas Luchner, Manfred Seeberger ${ }^{6}$, Christian Mueller6, Christoph Liebetrau ${ }^{3}$, \\ Svetlana Apostolovic ${ }^{7}$, Radmilo Jankovic ${ }^{7}$, Dragic Bankovic ${ }^{8}$, Marina Jovic ${ }^{8}$, \\ Vesselin Mitrovic ${ }^{3}$, Holger Nef, ${ }^{3,9}$, Helge Mollmann'3, Karl Werdan', \\ Christian W. Hamm ${ }^{3,9}$, Axel Schlitt ${ }^{1,10}$ \\ ${ }^{1}$ Department of Medicine III, University Clinic Halle (Saale), Halle, Germany \\ ${ }^{2}$ Department of Medicine, Clinic Grabs, Grabs, Switzerland \\ ${ }^{3}$ Department of Cardiology, Kerckhoff Heart and Thorax Center, Bad Nauheim, Germany \\ ${ }^{4}$ Department of Medicine II, Clinic Groß-Umstadt, Groß-Umstadt, Germany \\ ${ }^{5}$ Department of Medicine II, University of Regensburg, Regensburg, Germany \\ ${ }^{6}$ Department of Cardiology, University of Basel, Basel, Switzerland \\ ${ }^{7}$ University Cardiology Clinic, Niš, Serbia \\ ${ }^{8}$ Department of Mathematical Sciences, University of Kragujevac, Kragujevac, Serbia \\ ${ }^{9}$ Department of Medicine I, University Clinic of Gießen and Marburg, Gießen, Germany \\ ${ }^{10}$ Department of Medicine I, Paracelsus-Harz-Clinic Bad Suderode, Quedlinburg, Germany \\ Email: ${ }^{*}$ TWolters@gmx.net
}

Received 2 July 2014; revised 17 August 2014; accepted 1 September 2014

Copyright (C) 2014 by authors and Scientific Research Publishing Inc.

This work is licensed under the Creative Commons Attribution International License (CC BY). http://creativecommons.org/licenses/by/4.0/

(c) (i) Open Access

\section{Abstract}

Aims: The value of ECG in the perioperative risk stratification under modern treatment options is uncertain. The main objective of the present analysis was to determine the usefulness of a preoperative ECG derivation for evaluating the risk of perioperative morbidity and mortality. Methods: We performed a secondary analysis of the prospective, international, multicenter, observational "No-Risk" Study (N-terminal B-type natriuretic peptide [NT-proBNP] for the assessment of the perioperative cardiac risk after major noncardiac surgery) to determine the prognostic value of 12lead ECG. Inclusion criteria were age $>55$ years and at least one of the following cardiovascular risk factors: arterial hypertension, diabetes mellitus, dyslipidemia, active smoking, and family history positive for coronary heart disease. The combined primary endpoint included total mortality, acute myocardial infarction (NSTEMI and STEMI), cardiopulmonary resuscitation, heart fail-

\footnotetext{
*Corresponding author.
}

How to cite this paper: Wolters, T., et al. (2014) Pathologic Q Waves and Prolonged QTc Time in Preoperative ECG Are Predictive for Perioperative Cardiovascular Events. World Journal of Cardiovascular Diseases, 4, 498-509.

http://dx.doi.org/10.4236/wjcd.2014.41060 
ure, and asystole or ventricular fibrillation during hospitalization. ECGs from 616 patients enrolled from 2006 to 2009 prior to noncardiac surgery in the No-Risk Study were analyzed. Results: The mean age was $67.6( \pm 8.1)$ years; $300(48.7 \%)$ patients were male. Fourteen $(2.3 \%)$ patients suffered from the combined primary endpoint while in the hospital. In Kaplan-Meier analyses, a pathologic $Q$ wave and QTc $>500$ ms were significantly related to the incidence of the primary endpoint $(p<0.001, p=0.042$, respectively), whereas other ECG parameters such as LBBB, RBBB, PQ interval, QRS interval, and others were not related to worse in-hospital outcome. Conclusion: The 12-lead ECG is still an important diagnostic tool for perioperative risk assessment of cardiovascular events in noncardiac surgery in patients at risk.

\section{Keywords}

\section{ECG, Perioperative Risk, Cardiovascular Risk}

\section{Introduction}

The number of patients who undergo noncardiac surgery per year is constantly increasing worldwide. Indeed, severe complications such as perioperative cardiac death, nonfatal cardiac infarction, or nonlethal cardiac arrest develop in about 500,000 to 900,000 of those patients [1]. Furthermore, perioperative cardiovascular complications develop in $3.9 \%$ of patients with cardiac risk factors in whom noncardiac surgery is performed [1], which are mostly related to (unknown) coronary heart disease (CHD) [2].

Some clinical indices (Goldman, Detsky, Lee) have been established for stratifying perioperative cardiovascular risk in noncardiac surgery [2]-[4]. Furthermore, numerous studies exist in which the additive predictive value of pathologic electrocardiographic findings is discussed. Cardiac markers, namely, brain natriuretic peptide (BNP), N-terminal brain type natriuretic peptide (NT-proBNP), and troponin (troponin I and T) also seem to be useful for risk stratification [5] [6].

Preoperative 12-lead ECG is considered to be an easy and economic screening tool; thus, it is used in the preoperative period at many centers to detect pathologic heart conditions, above all asymptomatic CHD which might not have been discovered in the patient yet and would require further diagnostic steps [7]. However, some studies claim that 12-lead ECG is an unsuitable screening tool for undetected CHD and that its predictive value regarding postoperative complications is low [8] [9].

\section{Methods}

\subsection{Patients}

We performed a secondary analysis of the prospective, international, multicenter, observational "No-Risk" Study (N-terminal B-type natriuretic peptide [NT-proBNP] for the assessment of the perioperative cardiac risk after major noncardiac surgery) to determine the prognostic value of 12-lead ECG. The study centers that contributed patient data to this subanalysis were: Department of Cardiology, Kerckhoff Heart and Thorax Center Bad Nauheim (Germany), Jung-Stilling Hospital Siegen (Germany), University Hospital Regensburg (Germany), University Hospital Halle/Saale (Germany), University Hospital Basel (Switzerland), University Hospital Valladolid (Spain), and University Hospital Kragujevac (Serbia).

From 2006 to 2009 a total number of 979 patients were enrolled prior to noncardiac surgery. Inclusion criteria were major noncardiac surgery under general anesthesia, age $>55$ years, and the presence of at least one of the following cardiovascular risk factors: arterial hypertension, diabetes mellitus, dyslipidemia, active smoking, and family history positive for coronary heart disease. Exclusion criteria were emergency surgery and inability to understand or to give written informed consent. All patients included in the study gave their written informed consent. The study was approved by the local ethical boards of the participating centers and has therefore been performed in accordance with the ethical standards laid down in the 1964 Declaration of Helsinki and its later amendments. Details of the No-Risk Study have been presented elsewhere [10]. 


\subsection{Clinical Endpoints}

The predefined combined primary endpoint included total mortality, acute myocardial infarction (NSTEMI and STEMI), cardiopulmonary resuscitation, heart failure, and asystole or ventricular fibrillation during the hospitalization period. Endpoint assessment was conducted according to the clinical standards of the respective centers. The diagnosis of peri- or postoperative myocardial infarction was made on the basis of the universal definition of myocardial infarction [11].

\subsection{ECG Analysis}

Preoperative electrocardiography was performed following local standards (paper speed 50 or $25 \mathrm{~mm} / \mathrm{s}$ ). The criteria for evaluating the preoperatively derived ECGs were determined before performing the analysis.

In patients in whom heart rate, PQ and QT time, and the duration of $\mathrm{P}$ wave, Q wave, and QRS complex were determined automatically, those parameters were manually verified and corrected if necessary. In the following the applied ECG criteria are described.

Atrial Fibrillation (AF): AF was diagnosed when fibrillation waves were detected instead of $\mathrm{P}$ waves. Further criteria for the definition of AF were a lack of an isoelectric line and RR intervals with a wide variety of RR intervals.

Preexcitation Syndrome: A PQ interval of less than $120 \mathrm{~ms}$ and possibly present delta waves defined ECG signs of preexcitation. A delta wave was considered to be a slow and premature rise in the R spike without an isoelectric line between the $\mathrm{P}$ wave and QRS complex.

AV Block: A PQ interval of more than 210 ms was defined as indicative for AV block I ${ }^{\circ}$. A progressive increase in that interval until one QRS complex was missing was taken as diagnosing an AV block $\mathrm{II}^{\circ}$ Mobitz type I. The AV block II $^{\circ}$ Mobitz type II is characterized by a sudden absence of the QRS complex without any prior progressive increase in the QT interval. If atrial excitation was not forwarded to the ventricles and a $\mathrm{P}$ wave was not followed regularly by a QRS complex, AV block III ${ }^{\circ}$ was diagnosed.

Electrical Heart Axis: In this analysis an electrical heart axis with a main vector between $-30^{\circ}$ and $90^{\circ}$ was considered physiologic; main vectors outside that range were classified as pathologic.

Ventricular Extrasystoles: Premature, prolonged, and mono- or polymorphic QRS complexes possibly with a compensatory pause constituted the electrocardiographic signs for ventricular extrasystoles.

Myocardial Hypertrophy: To diagnose left ventricular hypertrophy, a Sokolow-Lyon index exceeding 3.4 $\mathrm{mV}$ was required (SV1/SV2 + RV5/RV6), for right ventricular hypertrophy an index higher than $1.05 \mathrm{mV}$ (RV1/RV2 + SV5/SV6).

Bundle Branch Block: A QRS interval of maximally 110 ms was considered to be normal. Any prolongation indicated a block of the ventricular conduction. In incomplete blocks, the QRS interval remains normal.

A complete left bundle branch block was defined as a QRS interval prolongation of more than $110 \mathrm{~ms}$, a broad $\mathrm{S}$ spike in $\mathrm{V}_{1}$, and broad, deformed $\mathrm{R}$ spikes in $\mathrm{I}, \mathrm{aVL}, \mathrm{V}_{5}$, and $\mathrm{V}_{6}$. An incomplete left bundle branch block was diagnosed when the typical deformation of the QRS complex but a normal QRS interval was found.

When partial left bundle branch blocks were considered, normal QRS interval and poor R wave progression in the chest leads were indicative. Furthermore, a left anterior fascicular block (LAFB) was characterized by a heart axis deviation to less than $-30^{\circ}$, prominent $S$ spikes in $V_{5}$ and $V_{6}$ and a small $Q$ wave in I and aVL. In the case of a left posterior fascicular block (LPFB), a heart axis deviation to more than $120^{\circ}$ and small Q spikes in II, III, and aVF were traced.

Right bundle branch block was defined by prolongation of the QRS interval, rSR' configuration in V1- and V2-QRS complexes and broad S in I, aVL, and V6.

Incomplete RBBB was defined by QRS duration between 110 and $120 \mathrm{~ms}$. Other criteria were the same as for complete RBBB.

Pathologic Q Waves: Signs of prior myocardial infarction were defined as pathologic Q waves (Pardée Q). Presence of pathologic $Q$ waves was diagnosed if the duration of the waves surpassed $30 \mathrm{~ms}$ and the amplitudes exceeded one third of the following RS complex in corresponding leads.

QTc Time: The QTc time was determined by applying Bazett's formula [12]. In this analysis, QTc > $500 \mathrm{~ms}$ was considered as clearly pathologic.

ST/T Changes: ST changes were considered abnormal if they were horizontal or descending depressions of at least $0.1 \mathrm{mV} 80 \mathrm{~ms}$ after the $\mathrm{J}$ point. A pathologic $\mathrm{T}$ wave was present if it was negative in any lead, except for 
aVR and V1. If the main vector of the respective lead was negative, the T wave was allowed to be negative, too.

\subsection{Statistics}

Statistical analysis was performed with SPSS $11.5^{\circledR}$. Arithmetical mean and standard deviation were used to describe continuous, symmetrically distributed variables. The $t$-test was employed to compare the arithmetic mean between two groups. Continuous variables with skewed distribution were characterized applying the median and $25 \%$ and $75 \%$ quartiles, respectively. For comparison of those variables, the Mann-Whitney U-test was chosen. Categorical variables were described in absolute numbers and percentages; for comparison between groups the Chi-Squared test was performed. Kaplan-Meier analyses with log-rank test were performed for endpoint analyses. For this, continuous variables were transformed into binary variables, applying the respective median. Differences in outcome between the groups were considered to be significant if the $p$ value was 0.05 or less.

\section{Results}

\subsection{Patient Characteristics}

The mean age of the patients was $67.6( \pm 8.1)$ years, and $300(48.7 \%)$ of them were male. Neither age nor sex differed significantly between the groups with and without combined primary endpoint (Table 1).

According to patient history, prior myocardial infarction was reported in 51 patients (8.3\%). Patients with primary endpoint had suffered from prior myocardial infarction more often than those patients without myocardial infarction in their medical history $(p=0.005)$ (Table 1$)$.

Table 1. Patient characteristics.

\begin{tabular}{|c|c|c|c|c|}
\hline Variable & Study population $(n=616)$ & No endpoint $(n=602)$ & With endpoint $(n=14)$ & $p$-value \\
\hline Age & $67.6( \pm 8.1)$ & $67.6( \pm 8.1)$ & $66.8( \pm 7.6)$ & 0.71 \\
\hline Male Sex & $300(48.7)$ & $292(48.5)$ & $8(57.1)$ & 0.523 \\
\hline BMI & $27.9( \pm 4.8)$ & $27.9( \pm 4.8)$ & $28.3( \pm 5.1)$ & 0.756 \\
\hline Arterial Hypertension & $521(84.6)$ & $512(85)$ & 9 (64.3) & 0.033 \\
\hline Dyslipidemia & $189(30.7)$ & $186(30.9)$ & $3(21.4)$ & 0.448 \\
\hline Diabetes Mellitus & $158(25.6)$ & $155(25.7)$ & $3(21.4)$ & 0.714 \\
\hline Positive Family History for CHD & $121(19.6)$ & 120 (19.9) & $1(7.1)$ & 0.234 \\
\hline Active Smoking & 147 (23.9) & $143(23.8)$ & $4(28.6)$ & 0.676 \\
\hline CHD & $86(14)$ & $82(13.6)$ & $4(28.6)$ & 0.111 \\
\hline CHD in Angiography & $40(6.5)$ & $38(6.3)$ & $2(14.3)$ & 0.231 \\
\hline Prior MI & $51(8.3)$ & $47(7.8)$ & $4(28.6)$ & 0.005 \\
\hline Prior PCI & $46(7.5)$ & $45(7.5)$ & $1(7.1)$ & 0.963 \\
\hline Prior ACB-OP & 24 (3.9) & $23(3.8)$ & $1(7.1)$ & 0.525 \\
\hline Prior Cerebral Infarction & $40(6.5)$ & $37(6.1)$ & $3(21.4)$ & 0.022 \\
\hline PAOD & $123(20)$ & $119(19.8)$ & $4(28.6)$ & 0.417 \\
\hline Renal Insufficiency & $53(8.6)$ & $52(8.6)$ & $1(7.1)$ & 0.844 \\
\hline Liver Disease & $20(3.2)$ & $19(3.2)$ & $1(7.1)$ & 0.405 \\
\hline Chronic Pulmonary Disease & $91(14.8)$ & $89(14.8)$ & $2(14.3)$ & 0.959 \\
\hline Malignant Disease & $149(24.2)$ & $141(23.4)$ & $8(57.1)$ & 0.004 \\
\hline
\end{tabular}

Continuous symmetrically distributed variables described as arithmetical mean ( \pm standard deviation); Categorical variables described as absolute numbers and percentage in brackets (\%), CHD = Coronary Heart Disease, MI = Myocardial Infarction, PCI = Percutaneous Coronary Intervention, $\mathrm{ACB}-\mathrm{OP}=$ Aortocoronary Bypass Operation, PAOD = Peripheral Artery Occlusive Disease, BMI = Body Mass Index in kg/m². 
Prior cerebral infarction was reported in the patient histories of 40 individuals (6.5\%). Three of the patients with primary endpoint (21.4\%) had suffered from such an event in the past in comparison to only $6.1 \%$ of patients without primary endpoint $(p=0.022)$ (Table 1$)$.

Furthermore, 149 (24.2\%) of the patients were suffering from a malignancy at study inclusion or had one in their history. Regarding the 14 patients who suffered from the combined primary endpoint, eight reported present or past malignant disease (57.1\%). In the group without primary endpoint $23.4 \%$ of patients fulfilled this criterion, resulting in a significant difference between the groups $(p=0.004)$ (Table 1).

The most frequently present risk factor in the study population was arterial hypertension (521 individuals, $84.6 \%$ of study population). In patients without combined endpoint arterial hypertension was present in $85 \%$. Among patients with the combined endpoint arterial hypertension was significantly less frequently known (64.3\%, $p=0.033)$ (Table 1$)$.

The other study inclusion criteria (dyslipidemia, diabetes mellitus, family history positive for CHD, and active smoking) did not reach significance in comparison between the groups (Table 1).

\subsection{Cardiovascular Medication, Vital Parameters, and Clinical Findings}

The cardiovascular medications being taken by the study population can be derived from Table 2 . There were no significant differences between the groups. Vital parameters and clinical findings were also not different in the comparison of both groups. However, patients with a higher Lee-Index (Revised Cardiac Index) were more likely to suffer from the combined primary endpoint (Table 2).

\subsection{Laboratory Parameters}

The arithmetic mean for hemoglobin in the study population was $13.61 \mathrm{~g} / \mathrm{dl}( \pm 1.73)$. Considering the two subgroups separately, a normal mean value persisted. However, for patients who experienced the primary endpoint, it was $1.38 \mathrm{~g} / \mathrm{dl}$ lower $(p=0.003$ ) (Table 3 ). The results concerning the predictive value of troponin T and NTproBNP in the No-Risk Study were published previously [10].

\section{Surgical Procedures}

Abdominal and general surgery procedures were performed in the majority of patients (217 [35.2\%]), followed by urological/gynecological (140 [22.7\%]) and vascular operations (129 [20.9\%]). Furthermore there were performed neurosurgical (42 [6.8\%]), orthopedic (37 [6\%]), thoracic (33 [5.4\%]), otorhinolaryngology and maxillofacial (16 [2.6\%]) and ophthalmic (2 [0.3\%]) operations.

\subsection{ECG Parameter}

The median of the electrocardiographically determined heart rate in this study population was 70 beats per minute. The difference in electrocardiographically determined heart rate was not significant between the groups of patients with and without occurrence of the combined primary endpoint $(74 / \mathrm{min}$ vs. $70 / \mathrm{min} ; p=0.193)$. In 446 (72.4\%) patients, electrocardiographic pathologies were present. The most frequently occurring electrocardiographic pathologies were presence of an electric heart axis deviation (107 [17.4\%]) and signs for myocardial hypertrophy (108 [17.5\%]). However, those variables did not reach significance in the between-groups comparison ( $p=0.758$ and $p=0.698$ respectively).

Atrial fibrillation was detected in 38 (6.2\%) patients. It was only diagnosed in 35 (5.8\%) patients without combined endpoint, whereas it was observed significantly more frequently in patients who experienced the endpoint (3 patients, [24.1\%], [ $p=0.016]$ ).

Electrocardiographic signs indicating prior myocardial infarction (Pardée Q in corresponding leads) were found in 54 patients (8.8\%). Among them, 49 (8.2\%) patients without and 5 (35.7\%) patients with primary endpoint presented with those kinds of ECG changes $(p<0.001)$. In the present analysis, a previous myocardial event could only be derived from the patient history in 16 (29.6\%) of 54 patients with electrocardiographic signs of prior myocardial infarction.

The mean value for the corrected QT time was $424 \mathrm{~ms}( \pm 32)$. In patients who suffered from the primary endpoint it was prolonged to $440 \mathrm{~ms}( \pm 39)(p=0.046)$. Of the 14 patients experiencing the primary endpoint, the QTc time was higher than the cut-off value $(500 \mathrm{~ms})$ in $14.3 \%$. In the group without the endpoint, such a prolongation was only found in $2.5 \%$ of the patients $(p=0.008)$ (Table 4$)$. 
Table 2. Medication, vital parameters, and clinical findings.

\begin{tabular}{|c|c|c|c|c|}
\hline Variable & Study population $(n=616)$ & No endpoint $(n=602)$ & With endpoint $(n=14)$ & $p$-value \\
\hline \multicolumn{5}{|l|}{ Medication $n$ (\%) } \\
\hline ASS and/or Clopidogrel & $224(36.4)$ & $218(36.2)$ & $6(42.9)$ & 0.609 \\
\hline Beta-Blocker & $272(44.2)$ & $269(44.7)$ & $3(21.4)$ & 0.083 \\
\hline ACE Inhibitor/ARB & $341(55.4)$ & $334(55.5)$ & $7(50)$ & 0.683 \\
\hline Diuretics & $152(24.7)$ & $149(24.8)$ & $3(21.4)$ & 0.776 \\
\hline Oral Anticoagulation & $43(7)$ & $42(7)$ & $1(7.1)$ & 0.981 \\
\hline \multicolumn{5}{|c|}{ Vital parameters and clinical findings at admission } \\
\hline RR systolic & $144.6( \pm 21.8)$ & $144.8( \pm 21.8)$ & $138.1( \pm 20.8)$ & 0.273 \\
\hline Cardiac Insufficiency & $32(5.2)$ & $31(5.1)$ & $1(7.1)$ & 0.740 \\
\hline NYHA Stage n (\%) & & & & 0.236 \\
\hline $\mathbf{0}$ & $287(46.6)$ & $283(47)$ & $4(28.6)$ & \\
\hline $\mathbf{I}$ & $187(30.4)$ & $183(30.4)$ & 4 (28.6) & \\
\hline II & $120(19.5)$ & 114 (18.9) & $6(42.9)$ & \\
\hline III & $21(3.2)$ & $21(3.5)$ & 0 & \\
\hline IV & $1(0.2)$ & $1(0.2)$ & 0 & \\
\hline CCS Stage n (\%) & & & & 0.542 \\
\hline $\mathbf{0}$ & $360(58.4)$ & 353 (58.6) & $7(50)$ & \\
\hline $\mathbf{I}$ & $189(30.7)$ & $185(30.7)$ & $4(28.6)$ & \\
\hline II & $62(10.1)$ & $59(9.8)$ & $3(21.4)$ & \\
\hline III & $5(0.8)$ & $5(0.8)$ & 0 & \\
\hline Revised Cardiac Risk I & & & & 0.035 \\
\hline $\mathbf{0}$ & $421(68.3)$ & $416(69.1)$ & $5(35.7)$ & \\
\hline 1 & $148(24)$ & $142(23.6)$ & $6(42.9)$ & \\
\hline 2 & $39(6.3)$ & $37(6.1)$ & $2(14.3)$ & \\
\hline 3 & $7(1.1)$ & $6(1)$ & $1(7.1)$ & \\
\hline 4 & $1(0.2)$ & $1(0.2)$ & 0 & \\
\hline
\end{tabular}

Continuous symmetrically distributed variables described as arithmetical mean ( \pm standard deviation); Categorical variables described as absolute numbers and percentage in brackets (\%); RR systolic = systolic blood pressure in mmHg; Pulse rate in beats per minute; Signs of cardiac insufficiency: peripheral edema/wet rale in auscultation/pleural effusion, ARB = Angiotensin Receptor Blocker, NYHA = New York Heart Association, CCS = Canadian Cardiovascular Society.

\section{Kaplan-Meier Analysis}

The variables that showed a significant difference in comparison between the groups were subjected to KaplanMeier analysis. Continuous variables were separated along the median and thus transformed to binary variables.

Preoperative NT-proBNP and Hs-TnT showed significant differences in the log-rank testing. The prognostic values of those parameters in the present study population have been extensively discussed in previous work [10].

In log-rank testing, a difference in prognosis for event-free survival was found for patients with or without a malignant disease $(p=0.036)$.

Atrial fibrillation in the preoperative ECG missed significance in log-rank testing $(p=0.078)$. Electrocardio- 
Table 3. Laboratory parameters.

\begin{tabular}{ccccc}
\hline Variable & Study population $(\boldsymbol{n}=\mathbf{6 1 6})$ & No endpoint $(\boldsymbol{n}=\mathbf{6 0 2})$ & With endpoint $(\boldsymbol{n}=\mathbf{1 4})$ & $\boldsymbol{p}$-value \\
\hline $\mathrm{Hb}$ & $13.61( \pm 1.73)$ & $13.65( \pm 1.7)$ & $12.27( \pm 2.53)$ & 0.003 \\
Creatinine & $0.88(0.75 / 1.06)$ & $0.97(0.75 / 1.06)$ & $0.99(0.8 / 1.31)$ & 0.213 \\
Leukocytes & $7.03(5.9 / 8.7)$ & $7.01(5.9 / 8.86)$ & $7.18(5.98 / 9.98)$ & 0.474 \\
Thrombocytes & $238(204 / 288)$ & $238(204 / 288)$ & $237(191 / 330)$ & 0.827 \\
HsTnT & $5(3 / 10)$ & $5(3 / 10)$ & $15(5 / 78)$ & $<0.001$ \\
NT-proBNP & $134.9(65.5 / 332.9)$ & $129.5(63 / 324.4)$ & $277.3(194.9 / 3088.3)$ & 0.003 \\
\hline
\end{tabular}

Continuous symmetrically distributed variables described as arithmetical mean ( \pm standard deviation); Continuous variables with skew distribution

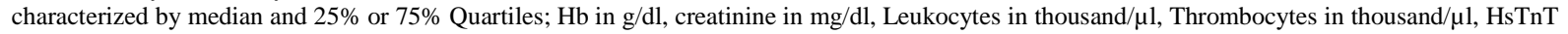
in $\mathrm{pg} / \mathrm{ml}$, NT-proBNP in pg/ml, $\mathrm{Hb}=$ Hemoglobin, HsTnT = high sensitive Troponin T, NT-proBNP = N-terminal brain type natriuretic peptide.

Table 4. ECG parameters in patients with and without occurrence of the combined primary endpoint.

\begin{tabular}{ccccc}
\hline ECG Parameter & Study population $(\boldsymbol{n}=\mathbf{6 1 6})$ & No endpoint $(\boldsymbol{n}=\mathbf{6 0 2})$ & With endpoint $(\boldsymbol{n}=\mathbf{1 4})$ & $\boldsymbol{p}$-value \\
\hline Heart Rate & $70(62 / 80)$ & $70(62 / 80)$ & $73.5(64 / 89.5)$ & 0.193 \\
Atrial Fibrillation & $38(6.2)$ & $35(5.8)$ & $3(24.1)$ & 0.016 \\
Preexcitation Syndrome & 3 & 3 & $1(7.1)$ & 0.921 \\
AV Block & $40(6.5)$ & $39(6.5)$ & $2(14.3)$ & 0.758 \\
Deviation of Electric Heart Axis & $107(17.4)$ & $105(17.4)$ & $1(7.1)$ & 0.554 \\
Ventricular Extrasystoles & $25(4.1)$ & $24(4)$ & $3(21.4)$ & 0.698 \\
Myocardial Hypertrophy & $108(17.5)$ & $105(17.4)$ & $1(7.1)$ & 0.297 \\
Bundle Branch Block & $112(18.7)$ & $49(8.2)$ & $5(35.7)$ & $<0.001$ \\
Pathologic Q Waves & $54(8.8)$ & $423.2( \pm 31.3)$ & $440.21( \pm 38.9)$ & 0.046 \\
QTc Time in ms & $423.6( \pm 31.5)$ & $15(2.5)$ & $2(14.3)$ & 0.008 \\
QTc $>$ 500 ms & $17(2.8)$ & $68(11.3)$ & $435(72.3)$ & $11(78.6)$ \\
ST/T Changes & $70(11.4)$ & $446(72.4)$ & 0.727 & 0.601 \\
\hline
\end{tabular}

Continuous symmetrically distributed variables described with arithmetical mean ( \pm standard deviation); Continuous variables with skew distribution characterized by median and $25 \%$ or $75 \%$ quartiles; Categorical variables described in absolute numbers and percentage in brackets (\%), heart rate in QRS complexes per minute.

graphic signs of prior myocardial infarction and a prolongation of the QTc time (dichotomized by a QTc time of $500 \mathrm{~ms}$ ) had a significant influence on shorter event-free survival ( $p<0.001$ and $p=0.042$, respectively) (Figure 1, Figure 2).

The combined endpoint was experienced by 14 individuals. Eleven of those individuals deceased. The causes of death and the frequency of the other endpoints are presented in Table 5.

\section{Discussion}

Perioperative cardiovascular morbidity and mortality represent the most relevant perioperative complications. An increased cardiovascular risk profile in the general population is responsible for the fact that cardiovascular complications in the perioperative setting have not decreased decisively in the past three decades [13].

According to current guidelines, routine derivation of preoperative ECG is only recommended for patients at risk [14]. Lee et al. [4] stated that individuals with pathologic Q waves in preoperative ECG (and thus a higher score on the revised cardiac index) require closer clinical attention without specifying which would be the next 


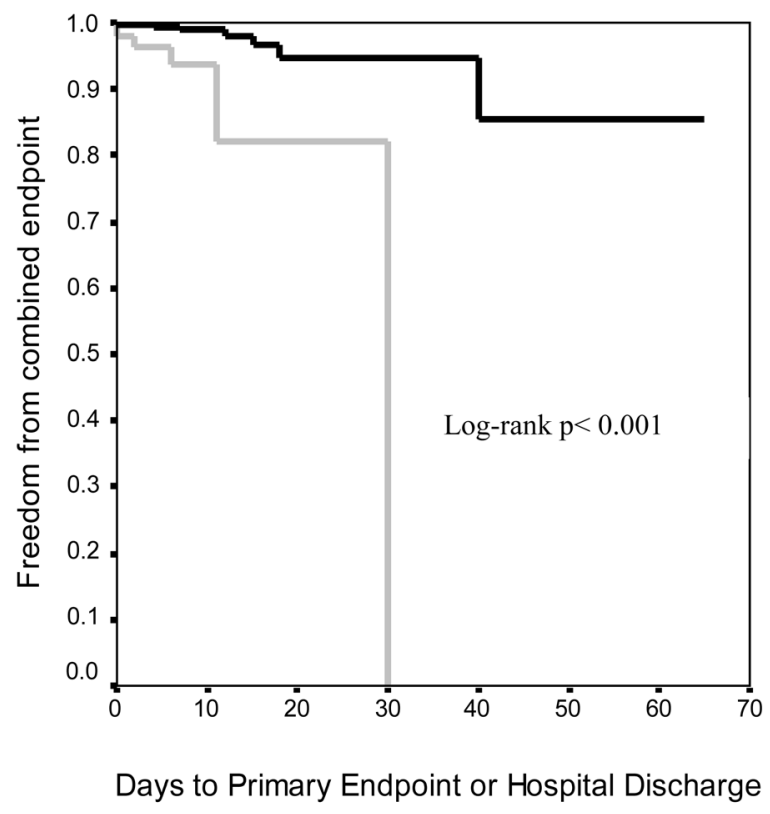

Figure 1. Kaplan-Meier Analyses with log-rank test: Freedom from combined endpoint after elective noncardiac surgery in patients with absence of pathologic Q-waves in preoperative ECG (black line) vs. presence of pathologic Q-waves in preoperative ECG (grey line).

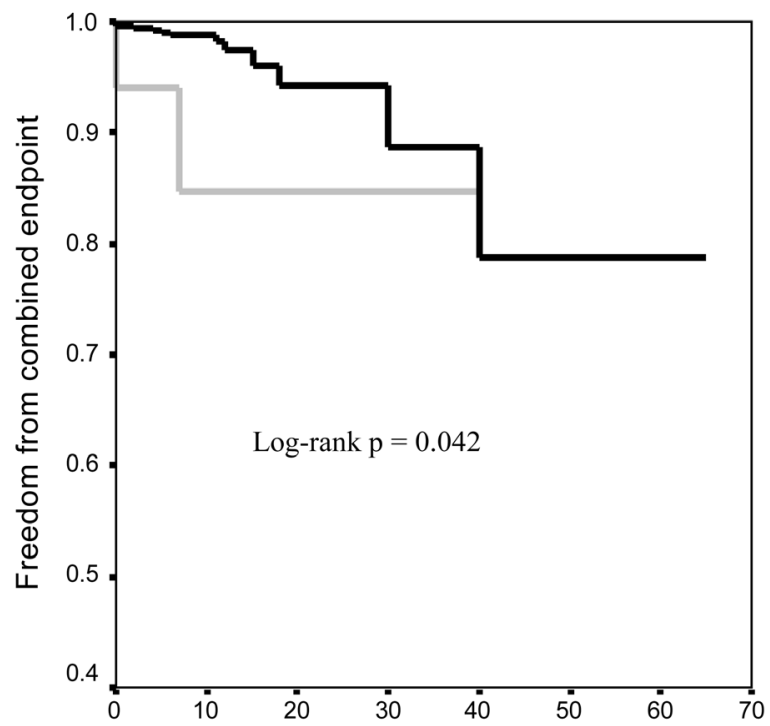

Days to Primary Endpoint or Hospital Discharge

Figure 2. Kaplan-Meier Analyses with log-rank test: Freedom from combined endpoint after elective noncardiac surgery in patients with QTc time $\leq$ $500 \mathrm{~ms}$ in preoperative ECG (black line) vs. > $500 \mathrm{~ms}$ in preoperative ECG (grey line).

step in preoperative diagnostics. Similarly Fleisher et al. [14] mentioned 12-lead resting ECG in their recommendations for preoperative risk stratification and suggest exercise stress testing as further preoperative diagnostic tool to estimate perioperative cardiac risk. The importance of routine preoperative 12-lead resting ECG in patients not in the defined risk groups is currently being controversially discussed in the literature. In this special context there is still a need for further and conclusive investigation [7]-[9] [15] [16]. 
Table 5. Combined primary endpoint.

\begin{tabular}{|c|c|c|}
\hline & Events & n (\%) \\
\hline & Patients suffering from combined primary endpoint & $14(2.3)$ \\
\hline \multirow{5}{*}{\multicolumn{2}{|c|}{ 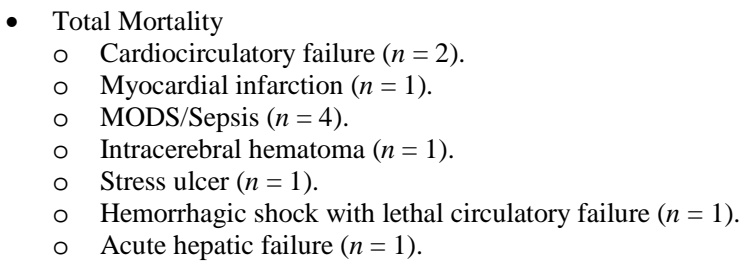 }} & \multirow{5}{*}{$11(1.8)$} \\
\hline & & \\
\hline & & \\
\hline & & \\
\hline & & \\
\hline \multicolumn{2}{|c|}{ - Asystole or ventricular fibrillation } & $6(1)$ \\
\hline \multicolumn{2}{|c|}{ - Cardiopulmonary resuscitation } & $3(0.5)$ \\
\hline \multicolumn{2}{|c|}{ - Acute decompensated cardiac insufficiency } & $2(0.3)$ \\
\hline \multicolumn{2}{|c|}{ - Acute myocardial infarction (NSTEMI und STEMI) } & $1(0.2)$ \\
\hline
\end{tabular}

MODS: Multi-organ dysfunction syndrome.

\subsection{ECG Parameters}

The main objective of the present analysis was to determine the usefulness of a preoperative ECG derivation for evaluating the risk of perioperative morbidity and mortality. In various studies different ECG parameters are considered to be relevant, resulting in a confusing variety of opinions about which parameters had prognostic importance [7]-[9] [15] [17] [18].

In our analysis at least one of the ECG pathologies which we took into account was found in $72.4 \%$ of our study subjects. However, pathologic ECGs as a total did not occur more frequently in patients with the primary endpoint than in those without (Table 4).

At $75.2 \%$, a comparably high percentage of pathologic ECGs were found in a study by Liu et al. [8]. Notably, three out of 14 patients (21\%) experiencing the primary endpoint in our analysis did not show any abnormal results in the preoperative ECG. The variables "pathologic Q waves" and "QTc more than 500 ms" reached significance in Kaplan-Meier analysis with log-rank testing (Figure 1, Figure 2). Atrial fibrillation, which was a significant predictor in between-group comparisons, missed significance in log-rank testing. In comparable studies, atrial fibrillation didn’t either reach statistical significance [7]-[9] [15] [17] [18].

Regarding the predictive value of "QTc time above 500 ms" Payne et al. [17] described similar results in their study population comprising 345 individuals. Furthermore, the findings of Biteker et al. [18] can also be supported by the present study. Biteker et al. also showed that preoperative QTc time was significantly longer for individuals experiencing perioperative cardiovascular events than for the rest of their study population. In addition to this, Biteker et al. found in a multivariate analysis that risk increased by $13 \%$ for every $10 \mathrm{~ms}$ of QTc prolongation [18].

The most common reasons for QTc alterations are medications [19]. In the context of the aforementioned observations preoperative ECG derivation could be a useful tool to detect QTc prolongation and thus those individuals potentially at an elevated cardiovascular risk. If an electrocardiographically detected QTc prolongation leads to a focused search for a responsible substance, it may help discover a reversible cause of the problem and consequently further minimize perioperative cardiovascular risk.

Presence of pathologic Q waves in the ECGs of this study population was significantly associated with shorter event-free survival than in individuals without such signs (Figure 1). Lee et al. [4] also traced a strong association between the presence of pathologic Q waves in preoperative ECG and perioperative cardiac complications. This criterion was consequently integrated into the Lee Index where it is defined as a sign of ischemic heart disease, thus adding one more point to the index.

At first glance it seems to be questionable as to whether an ECG is necessary to find out about prior myocardial infarction because such an event often is symptomatic and can thus be derived from the patient history. However, the prevalence of silent myocardial infarction increases with age up to $6.4 \%$ in the general population 
[20], in diabetic patients even up to 34\% [21]. Unrecognized or silent myocardial infarction should not be considered to be minor events [22]. In the Framingham study, it was shown that prognosis regarding another cardiac event after silent infarction is similar to the prognosis after symptomatic heart attack [23].

Aronow deduced that all patients of at least 60 years of age should undergo frequent routine ECG derivation, especially before and after surgery, in order to possibly initiate protective pharmacotherapy [24]. De Torbal et al. [22] even suggest frequent ECG screening beginning with 55 years of age.

In the present analysis, a previous myocardial event could only be derived from the patient history in 16 (29.6\%) of 54 patients with electrocardiographic signs of prior myocardial infarction (pathologic Q waves). In the remaining 38 (70.4\%) of the patients, silent or untypical myocardial infarction might have occurred. Without preoperative ECG an important factor for perioperative risk assessment would have been missed in those individuals. Consequently, the results of our analysis underscore the findings postulated by Aronow and de Torbal.

The risk for silent myocardial infarction rises with cardiovascular risk factors such as arterial hypertension, diabetes mellitus, advanced age, and known cardiovascular diseases [25]. Considering the demographic development in most industrialized countries and a continuous increase in the prevalence of the aforementioned diseases, a further increase in the incidence of silent myocardial infarction must be expected. Furthermore, it should be taken into consideration that if an infarction is suspected in the perioperative setting, a preoperatively derived ECG could help to distinguish new from preexisting ECG abnormalities [15].

\subsection{Study Limitations}

The main limitation of our study is the low incidence of endpoints. Moreover, although the No-Risk study included 979 patients only in 616 cases an ECG was available. However, in our opinion the statistical power of our study is high enough to support the demand for routine preoperative ECG derivation in patients at risk.

\section{Conclusions}

We conclude from our analyses that first in case of QTc-prolongation it is necessary to identify medication underlying this phenomenon or even postpone the surgery. Second, in presence of pathological Q waves a more thorough preoperative cardiological examination, which may lead to optimization of the medication or even cardiac catheterization, should be taken into consideration.

Twelve-lead ECG is still important in the assessment of perioperative risk today, also with respect to state-ofthe-art surgical, anesthesialogical, and intensive care therapy.

\section{Funding}

This work was supported by Roche Diagnostics, Rotkreuz, Switzerland.

\section{Conflict of Interest}

On behalf of all authors, the corresponding author states that there is no conflict of interest.

\section{References}

[1] Devereaux, P.J., Goldman, L., Cook, D.J., Gilbert, K., Leslie, K. and Guyatt, G.H. (2005) Perioperative Cardiac Events in Patients Undergoing Noncardiac Surgery: A Review of the Magnitude of the Problem, the Pathophysiology of the Events and Methods to Estimate and Communicate Risk. Canadian Medical Association Journal, 173, 627-634. http://dx.doi.org/10.1503/cmaj.050011

[2] Goldman, L., Caldera, D.L., Nussbaum, S.R., Southwick, F.S., Krogstad, D., et al. (1977) Multifactorial Index of Cardiac Risk in Noncardiac Surgical Procedures. The New England Journal of Medicine, 297, 845-850. http://dx.doi.org/10.1056/NEJM197710202971601

[3] Detsky, A.S., Abrams, H.B., Forbath, N., Scott, J.G. and Hilliard, J.R. (1986) Cardiac Assessment for Patients Undergoing Noncardiac Surgery. A multifactorial clinical risk index. Archives of Internal Medicine, 146, 2131-2134. http://dx.doi.org/10.1001/archinte.1986.00360230047007

[4] Lee, T.H., Marcantonio, E.R., Mangione, C.M., Thomas, E.J., Polanczyk, C.A., Cook, E.F., et al. (1999) Derivation and Prospective Validation of a Simple Index for Prediction of Cardiac Risk of Major Noncardiac Surgery. Circulation, 100, 1043-1049. http://dx.doi.org/10.1161/01.CIR.100.10.1043 
[5] Karthikeyan, G., Moncur, R.A., Levine, O., Heels-Ansdell, D., Chan, M.T.V. and Alonso-Coello, P. (2009) Is a Preoperative Brain Natriuretic Peptide or N-Terminal Pro-B-Type Natriuretic Peptide Measurement an Independent Predictor of Adverse Cardiovascular Outcomes within 30 days of Noncardiac Surgery? A Systematic Review and Meta-Analysis of Observational Studies. Journal of the American College of Cardiology, 54, 1599-1606. http://dx.doi.org/10.1016/j.jacc.2009.06.028

[6] Omland, T., de Lemos, J.A., Sabatine, M.S., Christophi, C.A., Murguia Rice, M., Jablonski, K.A., et al. (2009) A Sensitive Cardiac Troponin T Assay in Stable Coronary Artery Disease. The New England Journal of Medicine, 361, 2538-2547. http://dx.doi.org/10.1056/NEJMoa0805299

[7] Correll, D.J., Hepner, D.L., Chang, C., Tsen, L., Hevelone, N.D. and Bader, A.M. (2009) Preoperative Electrocardiograms: Patient Factors Predictive of Abnormalities. Anesthesiology, 110, 1217-1222. http://dx.doi.org/10.1097/ALN.0b013e31819fb139

[8] Liu, L.L., Dzankic, S. and Leung, J.M. (2002) Preoperative Electrocardiogram Abnormalities Do Not Predict Postoperative Cardiac Complications in Geriatric Surgical Patients. Journal of the American Geriatrics Society, 50, 1186-1191. http://dx.doi.org/10.1046/j.1532-5415.2002.t01-1-50303.x

[9] van Klei, W.A., Bryson, G.L., Yang, H., Kalkman, C.J., Wells, G.A. and Beattie, W.S. (2007) The Value of Routine Preoperative Electrocardiography in Predicting Myocardial Infarction after Noncardiac Surgery. Annals of Surgery, 246, 165-170. http://dx.doi.org/10.1097/01.sla.0000261737.62514.63

[10] Weber, M., Luchner, A., Seeberger, M., Mueller, C., Liebetrau, C., Schlitt, A., et al. (2012) Incremental Value of High-Sensitive Troponin $\mathrm{T}$ in Addition to the Revised Cardiac Index for Peri-Operative Risk Stratification in NonCardiac Surgery. European Heart Journal, 11, 853-862.

[11] Thygesen, K., Alpert, J.S., White, H.D., Jaffe, A.S., Apple, F.S., Galvani, M., et al. (2007) Joint ESC/ACCF/AHA/ WHF Task Force for the Redefinition of Myocardial Infarction. Universal definition of Myocardial Infarction. European Heart Journal, 28, 2525-2538. http://dx.doi.org/10.1093/eurheartj/ehm355

[12] Bazett, H.C. (1997) An Analysis of the Time-Relations of Electrocardiograms. Annals of Noninvasive Electrocardiology, 2, 177-194. http://dx.doi.org/10.1111/j.1542-474X.1997.tb00325.X

[13] Yu, P.C., Calderaro, D., Gualandro, Marques, A.C., Pastana, A.F., Prandini, J.C., et al. (2010) Non-Cardiac Surgery in Developing Countries: Epidemiological Aspects and Economical Opportunities-The Case of Brazil. PLoS ONE, 5, e10607. http://dx.doi.org/10.1371/journal.pone.0010607

[14] Fleisher, L.A., Beckman, J.A., Brown, K.A., Calkins, H., Chaikof, E., Fleischmann, K.E., et al. (2008) ACC/AHA 2007 Guidelines on Perioperative Cardiovascular Evaluation and Care for Noncardiac Surgery: Executive Summary: A Report of the American College of Cardiology/American Heart Association Task Force on Practice Guidelines (Writing Committee to Revise the 2002 Guidelines on Perioperative Cardiovascular Evaluation for Noncardiac Surgery). Anesthesia \& Analgesia, 106, 685-712. http://dx.doi.org/10.1213/01/ane.0000309024.28586.70

[15] Noordzij, P.G., Boersma, E., Bax, J.J., Feringa, H.H., Schreiner, F., Schouten, O., et al. (2006) Prognostic Value of Routine Preoperative Electrocardiography in Patients Undergoing Noncardiac Surgery. American Journal of Cardiology, 97, 1103-1106. http://dx.doi.org/10.1016/j.amjcard.2005.10.058

[16] Fritsch, G., Flamm, M., Hepner, D.L., Panisch, S., Seer, J. and Soennichsen, A. (2011) Abnormal Pre-Operative Tests, Pathologic Findings of Medical History, and Their Predictive Value for Perioperative Complications. Acta Anaesthesiologica Scandinavica, 3, 339-350.

[17] Payne, C.J., Payne, A.R., Gibson, S.C., Jardine, A.G., Berry, C. and Kingsmore, D.B. (2011) Is There Still a Role for Preoperative 12-Lead Electrocardiography? World Journal of Surgery, 35, 2611-2616. http://dx.doi.org/10.1007/s00268-011-1289-y

[18] Biteker, M., Duman, D. and Tekkeşin, A.I. (2012) Predictive Value of Preoperative Electrocardiography for Perioperative Cardiovascular Outcomes in Patients Undergoing Noncardiac, Nonvascular Surgery. Clinical Cardiology, 35, 494499.

[19] Yap, Y.G. and Camm, A.J. (2003) Drug Induced QT Prolongation and Torsades de Pointes. Heart, 89, $1363-1372$. http://dx.doi.org/10.1136/heart.89.11.1363

[20] Nadelmann, J., Frishman, W.H., Ooi, W.L., Tepper, D., Greenberg, S., Guzik, H., et al. (1990) Prevalence, Incidence and Prognosis of Recognized and Unrecognized Myocardial Infarction in Persons Aged 75 Years or Older: The Bronx Aging Study. The American Journal of Cardiology, 66, 533-537. http://dx.doi.org/10.1016/0002-9149(90)90477-I

[21] Feringa, H.H., Karagiannis, S.E., Vidakovic, R., Elhendy, A., Cate, F.J., Noordzij, P.G., et al. (2007) The Prevalence and Prognosis of Unrecognized Myocardial Infarction and Silent Myocardial Ischemia in Patients Undergoing Major Vascular Surgery. Coronary Artery Disease, 18, 571-576. http://dx.doi.org/10.1097/MCA.0b013e3282f08e86

[22] de Torbal, A., Boersma, E., Kors, J.A., van Herpen, G., Deckers, J.W., van der Kuip, D.A., et al. (2006) Incidence of Recognized and Unrecognized Myocardial Infarction in Men and Women Aged 55 and Older: The Rotterdam Study. 
European Heart Journal, 27, 729-736. http://dx.doi.org/10.1093/eurheartj/ehi707

[23] Kannel, W.B. and Abbott, R.D. (1984) Incidence and Prognosis of Unrecognized Myocardial Infarction. An Update on the Framingham Study. The New England Journal of Medicine, 311, 1144-1147. http://dx.doi.org/10.1056/nejm198411013111802

[24] Aronow, W.S. (2003) Silent MI. Prevalence and Prognosis in Older Patients Diagnosed by Routine Electrocardiograms. Geriatrics, 58, 24-40.

[25] Valensi, P., Lorgis, L. and Cottin, Y. (2011) Prevalence, Incidence, Predictive Factors and Prognosis of Silent Myocardial Infarction: A Review of the Literature. Archives of Cardiovascular Diseases, 104, 178-188. http://dx.doi.org/10.1016/j.acvd.2010.11.013 
Scientific Research Publishing (SCIRP) is one of the largest Open Access journal publishers. It is currently publishing more than 200 open access, online, peer-reviewed journals covering a wide range of academic disciplines. SCIRP serves the worldwide academic communities and contributes to the progress and application of science with its publication.

Other selected journals from SCIRP are listed as below. Submit your manuscript to us via either submit@scirp.org or Online Submission Portal.
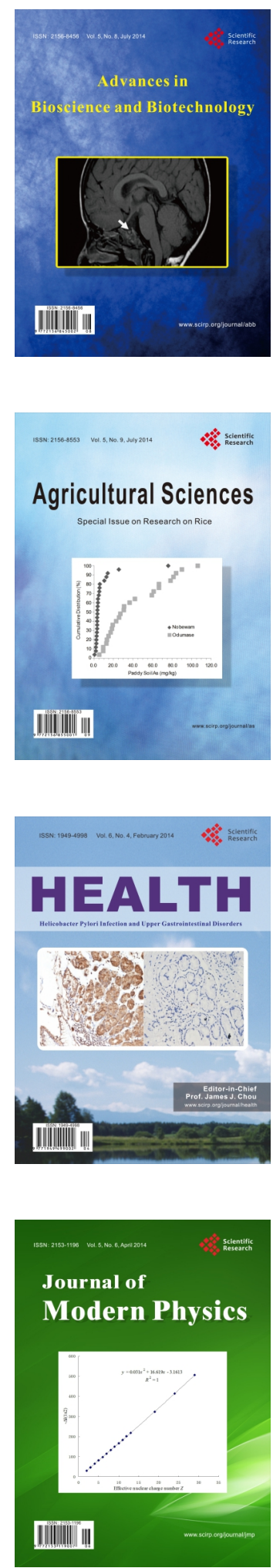
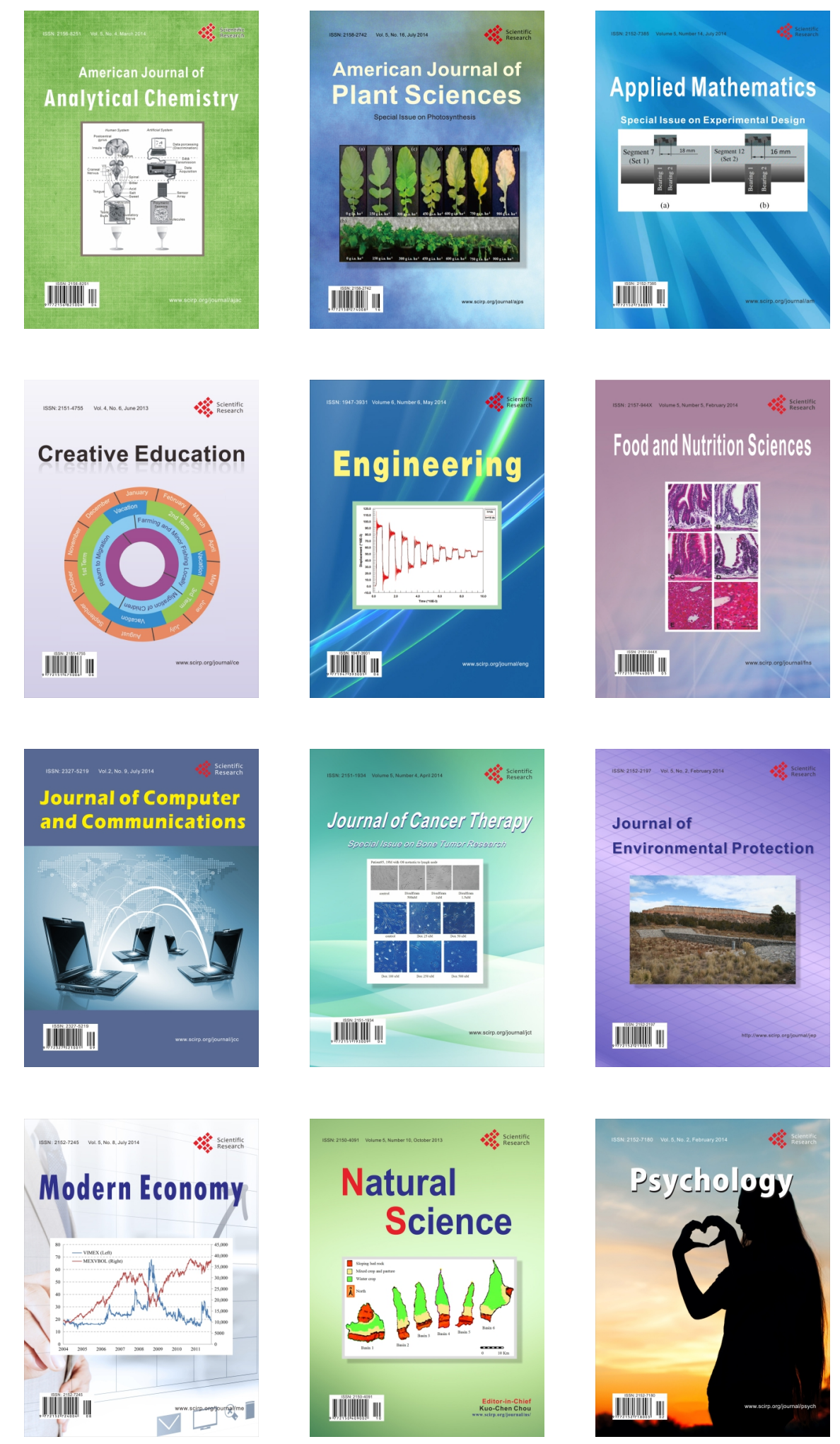